\title{
UN DOCUMENT DE RAVENNA DEL SEGLE X A BARCELONA
}

\author{
Anscari M. Mundó
}

A la tardor del 1978 vaig poder examinar un feix de fulls de pergamí provinents de relligadures i conservats a la secció de manuscrits de la Biblioteca de Catalunya'. Amb molta sorpresa, 1'esguard se'm va fixar en dos grans trossos de pergamí coberts d'una escriptura ben diferent de la nostra carolina catalana habitual. Vaig veure de seguida que es tractava d'una cursiva del nord d'Itàlia. En un primer intent de lectura aparegué clar el topònim Ravenne, i també el fet que ambdós trossos pertanyien al mateix document. La raresa d'aquesta peça d'arxiu m'ha semblat que podia servir per a homenatjar el doctor Frederic Udina, que ha estat el meu superior, és ara col-lega i serà sempre un bon amic.

Els documents de Ravenna són força cèlebres per l'existència d'un bon grup de papirs conservats com per miracle. Els escrits damunt de pergamí, encara que més tardans i abundosos, no són pas menys recercats. En qualsevol cas, creuria que aquest document retrobat a Barcelona és l'únic de la seva mena existent a la península ibèrica. La signatura actual és: Barcelona, Biblioteca de Catalunya, 2542 de la secció de còdexs.

I Dec a l'amabilitat del seu conservador, Dr. Amadeu J. Soberanas, la possiblitat d'aquesta troballa i de l'estudi consegüent; li ho agraeixo des d'aquí. 
La seva procedència immediata és la col-lecció de l'estudiós Manuel de Bofarull i Sartorio (1816-1892), que fou adquirida al seu fill, Francesc de Bofarull i Sans (1843-1938), l'any 1911, per l'Institut d'Estudis Catalans per a la seva Biblioteca?

No és fàcil saber d'on el va treure Manuel de Bofarull; en tot cas, és ben clar que fou desenganxat d'una relligadura de posts de fusta d'un còdex escrit a Itàlia, ara desconegut; es dedueix, però, de les restes de fusta enganxades al verso del document i també de les probationes penna que és de la fi del segle XII. Les més notables d'aquestes diuen: «ego valentinus debeo c raimundo aldeberg mallus» (?); "ego raimundus debeo X. solidis uxori cassii du intrante aprili» (repetit en part); «fuit//punator» «aue maria»; els noms de Valentino, Aldeberg i Cassio i el «du intrante aprile» són certament italians. A l'altre tros de pergamí apareixen aquestes altres probationes de les mateixes mans, pel que sembla: «in terra eg[i]pti sanctificanini» (!), repetit en part tres vegades; «primogeniti/santificamini//primogeniti Leui (?) quiic qui quiq (?)// quia dominus//mansa", que semblaria que indiquen la pertinença a un còdex bíblic. Aquestes notetes es troben escampades i separades en diverses posicions.

Els dos trossos del document fan $210 \times 292$ i $206 \times 292$ mm, respectivament, de màxima. És una mica difícil precisar les mides originals; si tenim en compte, però, el text mancant i els marges, ara desapareguts, es podria calcular un original d'aproximadament $427 \times 307 \mathrm{~mm}$. Actualment han estat muntats i relligats degudament.

L'estat de conservació del pergamí és bo i el text llegible, si se n'exceptuen les parts retallades que, com s'ha vist per les mides, afecten els quatre marges i la tira d'entremig dels dos trossos conservats, amb pèrdues de text en tots els casos.

L'escriptura és una bella cursiva nova itàlica del tipus en ús a Ravenna, derivat de la comuna romana del Baix Imperi. En canvi, les firmes autògrafes dels testimonis són en una carolina força rústega, de lletres més petites i estretes que les del text, i de línies mal traçades, especialment la del tercer testimoni.

${ }^{2}$ Al registre d'entrades de llibres, f. $6 v-8$. 
La transcripció que n'he fet - tot tenint en compte l'època del document i la claredat de l'escriptura, que no ofereix dificultats insuperables - és diplomàtica, no pas paleogràfica; d'aquí que hi he desfet les abreviatures comunes i només van entre parèntesi aquelles que podrien oferir algun dubte.

També n'ha estat conservada naturalment la grafia, puix que ofereix peculiaritats curioses per al filoleg. Així, per exemple: ha $(=a, 2,12)$; hac $(=a c, 6) ;$ huna $(7$, bis); husum $(8,21)$; haram $(8)$; halia (12); hetiam (14); hamodo (15); per contra: orto $(8,10)$; uic $(35,36,37)$; mahinationis (25). - patifico (2); Lutianus $(3,34)$; tirca (=circa, 7); fatiendi (15); iuditium (24); atimpleuerimus (28); ditimus (29). $\rightarrow$ seo $(=\operatorname{seu}, 4,5,31) ;$ da $(=$ de, 5$)$; ouenit $(6$, ouenisset 7); exucessionem (6, 17); occansionem (23); transfersimus (5, transfersionis 4, 31); surexerit (26); painam (37). No cal dir que tampoc no s'esmena la manca gairebé total de concordança sintàctica i de flexió nominal i verbal, etc.

La comparació amb altres documents ravennesos contemporanis ${ }^{3}$ ha permès d'emplenar alguns buits deixats en el text a causa dels retalls del pergamí, tal com ja he indicat. Per les signatures dels actors i testimonis del final del document es dedueix ben clar que les línies del text han estat molt més retallades de la banda dreta que de l'esquerra, on només deuen mancar entre 4 i 8 lletres. El nom del notari o tabellio ha desaparegut amb aquestes retallades. La comparació, però, amb les escriptures reproduïdes en alguns repertoris paleogràfics 4 permet de precisar la datació de l'escriptura del document conservat a Barcelona.

${ }^{3}$ He tingut a mà: M. FANTUZzI, Monumenti Ravennati dei secoli di mezzo, 6 vols., Venezia 1801-1804; A. TARLAZZI, Appendice ai Monumenti Ravennati, 2 vols., Roma 1869-1884; G. MARINI, I papiri diplomatici raccolti ed illustrati, Roma 1805: V. FEDERICI, Regesto di Sant Apollinare Nuovo, Roma 1907: V. FEDerici e G. Buzzi, Regesto della Chiesa di Ravenna. Le carte dell' Archivio Estense, 2 vols, Roma 1911-1931; H. MARUcCH, Monumenta papyracea latina Bibliothecae Vaticana, Romæ 1895.

4 Per exemple, «Archivo paleografico italianow III, 1884, pp. 85-86 (del 959); pp. 74-81 (del 973); ibíd., VII pl. 39 (964 cf. FANTUZZI I, 163-165, núm. xxxXI); VII pl. 41 (del 967); VII pl, 43 (del 970), cf. M. Prot et A. DE Boúard, Manuel de Paléographie latine et française, Album, Paris 1924, pl. II, 1 (del 970). 
Pel que fa al seu contingut, es pot resumir així: Pere, fill de Llucià, difunt, i la seva muller Donda, dita Bona, venen a Maria, filla de..., uns béns que posseeixen i que pervenien a Pere de la seva mare Petrònia. Aquests béns són: un cup (cupa) amb les seves parets a l'entorn, amb tot el que hi ha al seu davant, una cort i un hort al costat, amb l'ús del pou i del pòrtic del davant. El conjunt es troba en aquesta ciutat de Ravenna, a la regió i a prop de la basílica del Salvador, amb els seus termes justos i certs, que són: d'un costat, la plaça pública que va cap a la porta de Sant Llorenç, amb la propietat del prevere Ravenni (de l'Església de Ravenna); del tercer costat... i del quart, amb l'altra plaça que ve del palau. «Lliurem tot això a vosaltres, per tal que en pugueu fer i en disposeu segons la vostra voluntat, excepte la pensió [o cens] anual que s'ha de donar a la Santa Església Romana». Tots aquests béns, els tenia Pere per successió hereditària de la seva mare Petrònia difunta i que va haver de condividir amb el seu germà (prevere). Segueixen les fórmules cautelars de dret amb llur pena annexa. El document, anomenat pagina, fou donat per escriure a un tabel-lió (notari) de Ravenna (de qui s'ignora el nom), i fou sotasignat pels vendors i pels testimonis, entre els quals hi ha un consol i dos fills del consol Andreu.

Seguia almenys una altra signatura que, per les traces que en queden, gosaria creure que fos la del tabel-lió.

Heus aquí el que queda del text:

Ravenna, 962.

I $[\ldots \ldots \ldots]$

2 [sitque] imperante [dom]n[o] piissimo perpetuo augusto Otone ha deo coronato patifico magno imperatore anno [... in Ita]lia anno primo $d[$ ie ... mensis ......]

3 [ind(ictione)] quinta Rauenna. Profitens profiteor ego Petrus filio quondam Lutianus et ego Donda que uocatur Bona iug[al(e) .......

4 uenditijonis seo et transfersionis et corporalis traditionis nostre paginam, te presente, in dei nomine Maria filia [......]

5 ...Juendimus seo et transfersimus et da nostra iwra nostroque dominium nostrisque filiis et heredibus alienamus in iura et domi[nio......]

6 ...Jd habere hac possidere uidemus; que michi supradicto Petrus ouenit exucessione quondam Petronia genitrice m[ea......] 
7 ... oluenisset. Id est cupa huna in integro cum muras suas tirca se, huna cum subiuncta sua ante se omnino [canna coperta cum curte (?)]

8 antelse et orto suo in integro iuxta ipsa curte et husum puteo et hara portico ante se, quod est similiter [.....]

9 omni]bus ad easdem pertinentibus constituta in hac ciuitate Rauenna, in regione et prope basilice Saluatori[s ......]

10 ... cum suis iustis et certis in terra finibus. Et in terra fines eiusdem cupa et subiuncta et curte et orto [......]

11 ... lest ab uno loco platea publica qui pergit a porta Sancti Laurentii, et ab alio loco possidente Rauenno presbietro s[ancte Rau(ennatis) Ec(c)l(esi)a, et a tertio loco .......

12 et Ja quarto loco halia platea qui decurrit ha palatio. Ut in omnibus liceat tibi inibi introire et preocc [upare .......]

13 ...Jmam et nostram corporalem traditionem, quam de nostris manibus in tuis tradimus in presentiam testium qu [......]

14 ... $/ s$ cupa $(m)$ quieto tramite habendi dominium et potestatem habeatis, teneatis, possideatis hetia $[m \ldots . . .1$

15 face]re quod uolueritis exinde fatiendi necnon innouandi et disponendi, salua annua pensione hamodol.......

16 .... $c$ est sanctam Romanam Ecclesiam. In quantum uobis exinde pertinuerint, $q($ ue $) m$ nos predicti iugalis habemus $[\ldots . .$.

17 hac $\}$ omnia ut superius legitur ouenit exucessionem hereditario nomine a quondam bone memorie Petronia genitrice mea [... ...]

18 .... $]$ [pres]b/itero et gle[erman]o meo pro diuisionem que inter nos diuisimus et partiuimus. Pro eo quia exind[de ......]

$19[\ldots \ldots \ldots\} \ldots \ldots[\ldots \ldots]$

20 ...Jecusatione ......... [.....] generare incontra te emtrice neque incontra tuis fill iis .......)

21 ...sub]iuncta sua omnino canna coperta, cum curte et orto et husum puteo et cum ara portico suo uel cum ingress [......]

22 sicut] superius legitur, qui est posita iuxta platea qui decurrit a palatio pro ex parte uel in totum numquam agimus $n[\ldots . .$.

23 ... olccupamus neque agentibus quod discindere audeamus per nullam occansionem neque ingenium, non per nos neque per [......]

24 .... as non adeund(um) iuditium, non supplicandum principibus neque per ecclesiasticam interpellationem aut per regallem potestatem ... (?) ....

25 quodllibet doli mahinationis ingenium neque per ullo modo ullamque rationem contraria quod sensus humanus as [... ...]

26 ...e elsse securi et quieti exinde maneatis in omnibus. Et si quo- 
quo tempore queuis personas hominum surexerit qui uoblis ... ...)

27 ... clalumnia generare uoluerit, nos uenditoris cum nostris heredibus tibi tuisque heredibus ab omni personas in omnibus stare et deflensare promittimus in perpetuum. Quod si, quod]

28 absit et] auertat diuina potentia et omnia que superius legitur non obseruauerimus uel atimpleuerimus, et contra hec ire uel ag/ere uel contendere uoluerimus ... per]

29 quollibet modo uel titulum, pro quibus iuratas uoce ditimus per deum omnipotentem sedemque sanctam Apostolicam et uitam supradictorum dominorum nostro/rum atestationem confirmamus ...J

30 qui]a daturi nos promittimus cum nostris heredibus tibi emtrice tuisque heredibus, ante omnem litis initium aut interpellationem, pene nomine a [uri ebrizo uncias(?) ... et post pene solu]-

31 tionem] hanc paginam professi, sponsionis et uenditionis seo transfersionis corporalis traditionis in sua fi/rmitatem ... Quam uero paginam ....

32 in Dei nolmine tabellio huius ciuitatis Rauenna scribendum rogauimus, in qua nos subter manus nostras propria firmauimus testibusque a nobis rogiltis obtulimus subscribendum eorumqueJ

33 pres]enssi eam tibi q(uoru)m contradidimus sub die et mense et indictione supradicta quinta Rauenna. ) ) )

34 Signum] ++ manibus nostris supra dictis Petrus filio quondam Lutianus et Donda que uocatur Bona, iugal(is) iamdicti uenditoris ad omnial...

$35 \mathrm{~S} .+\ldots$ us consule uic paginam uindicionis, tradicionis de omnibus ut superius legitur facta in Maria suisque heredibus ad supradicti iugale rogatus testis set supradicto precio dante et acipientle...

$36 \mathrm{~S} .+$...filius] $q($ uon $) d(\mathrm{am})$ Andreas consule et negociatore wic pagina uindicionis, tradicionis de omnibus ut superius legitur facta in Maria suisque heredibus ad supradicti iugali rlolg[a]tus test [is ...

37 S.+ ...Jus filio conda Andrea consule et negociatore uic painam u/i]ndicionis, tradicionis de omnibus ut superius legitur fia)cta in Maria suisque h[eredibus ............] uin/dicionis, [tra]d[icionis] d[e ......]

39 $[\ldots \ldots \ldots \ldots]$

1.lin.prima integre excissa disparuit. -2. dubium an inter anno et in Italia aliz adfuerint litterae. -7. hun'bis scriptum. 11 . ste.rav.eccle ut coniicio ex documentis coævis. -16. habemus 
quamvis potius habenus videatur scriptum. -18 . ...]o [pres]b[itero] $b$ hastam habet transversam sicut pbro. lin.11: [et gle (rman) 0 meo de quibus aliqua elementa servantur. -19 . hastæ quattuor vel quinque litterarum remanent. -25. syllaba as ultima fortasse ar legenda. -31. professi ita scripsit notarius loco professionis. -33. [pres] enssi ita scriptum: q[uoru]m an q(uora $m$ ? ; signum ) ter scriptum et protractum usque in finem lineae. -36, utrum post nomen: filius (?)]q(uon)d(am) Andreas legendum sit, an forte: $q($ (uod) d(icitur) Andreas absque filius ? -37 . undicionis scripsit. -38 . hastæe et vestigia harum vocum restant sufficientia, -39. desunt linex fortasse aliorum subscriptorum nomina et certe notarii seu tabellionis afferentes.

Pel que es diu el text, «in regione et prope basilice Salvatoris» i «platea qui decurrit ha palatio», és clar que els béns venuts es trobaven als voltans de la basílica de Sant'Apollinare Nuovo i del palau anomenat de Teodoric. Els especialistes de la topografia ravennat podran, sens dubte, precisar encara més 5 .

Encara que establir l'època del document no ofereix dificultats - ja que l'escriptura, la llengua i el formulari apunten cap al segle $\mathrm{X}_{-}$, el retallat del pergamí, que féu desaparèixer la primera línia i part de la segona, no permet llegir-hi el nom del papa regnant i, amb prou feines, el de l'emperador.

De la segona línia se'n pot llegir força tros: algunes paraules, amb seguretat, d'altres resulten clares en fer la comparacio amb els formularis de documents coetanis de la regió ${ }^{6}$. No tinc cap dubte sobre el

${ }^{5}$ Només he pogut consultar: L.A. MURATORI, Rerum italicarum scriptores, I. 2, pp. 527 ss.: Spicilegium Ravennatis historio sive Monumenta historica ad ecclesiam et urbem Ravennatem spectantia; P. LUTHER, Rom und Ravenna bis zum 9. Jahrhundert, Berlin 1889; G. BuzzI, «La curia arcivescovile e la curia cittadina di Revenna dall' 850 al 1118 *, Bollenino dell' 'stituo Storico haliano 35, 1915; F. W. Deichmann, Ravenna: Geschichte und Monumenta, Wiesbaden 1969; A. AGNELLUS, Liber pontificalis ecclesia Ravennatis I, Bologna 1924; encara útil la vella edició de BETHMANN-WAITZ MGH, Script, rerum langobardicarum et italicarum, 1878, pp. 265 ss.: B. BISCHOFF und W. KOEHLER, «Eine illustrierte Aufgabe der spätantiken Ravennater Annalens, Medieval studies in memory of A.K. Porter I, Cambrigde, Mass. 1939, pp. 125-139.

6 Utilitzo les edicions de diplomataris de FANTUzzI (Monumenti), FEDERICI (Apollinare) i FEDERICI-BUZZI (Estense), citats a la nota 3, il'Archivio paleografico italiano i PROU-DE BOUARD, Manuel, citats a la nota 4, amb les dates corresponents. 
nom de l'emperador Otone, ja que les restes romases de les lletres no permeten cap altra lectura. En canvi, de la fórmula que inclou la data cronològica no se n'ha conservat prou per esvair els dubtes. Es llegeix bé: «ha deo coronato, pacifico, magno imperatore anno...», seguit de restes d'unes deu lletres tallades, entre les quals es veu «[Ita]lia», i després «anno primo», La repetició d'anno podria fer semblar que s'hi utilitzaven dues cronologies d'Otó. De fet, en els documents de Ravenna contemporanis seus ${ }^{7}$, es troba la doble menció d'anno, que emmarca la fórmula d'entremig: regni pietatis eius in Italia vero, seguida de l'any amb el numeral. Malgrat això, aquesta llarga fórmula de 29 lletres no es pot suposar aquí, car l'espai que es veu en el pergamí només en permet 10 i, entre aquestes, són certes, com he dit, les d'ltalia; si no és que s'hi vulguin suposar abreviatures que, però, em semblen forçades, almenys pel mot [pietatis ] irreduïble ${ }^{8}$.

7 De FANTUZzI, I p. 158 (del 953); p. 160 (964.I.15); p. 162 (964.II.4); p. 166 (964.IV.22); p. 168 (965.VIII.7); p. 169-170 (966.III.14), p. 172 (970.IV.8); vol. II, p. 27 (967.IV.7); p. 30 (967.X.12); vol, III, p. 5 (965.V.27); vol VI, p. 14 (962.V.14); especialment, id, I p. 163-165 (964.III.5) reproduït a l'Archivio paleografico italiano VII, pl. 39; del mateix Archivio VII, pl. 41 (del 967.XI); pl. 43 (970.VII.25); de FEDERICI-BUZZI, Estense (896.IX.8); PROU-DE BOUARD, Manuel pl. II, 1 (970); FEDERICI, Apollinare, (973.V.11); (977.I.9); (977.VII.30); (982 ...14); (983.VII.16); (983.VIII.6); (1000.I.16).

8 Les més semblants a fes fórmules del preàmbul i datació crònica i tòpica del document conservat a BArcelona són els ja citats, FANTuzzi, 1, p. 158: $\alpha$ Sitque imperante domuo Ottone a Deo coronato, pacifico magno imperatore in Italia anno tercio...» (del 963); id., I, p. 168: «...Ononi...magno imperatore anno regni pietatis in Italia anno quarto.... (del 965); id., II, p. 27: «... Ottone... pacifico magno imperatore anno regni pietatis eius in Italia vero anno sexto.... (del 967); id., vI, p. 14: *...Ottone... pacifico magno imperatore anno regni pietatis eius in Italia vero anno primo...» (del 962, de Forlf): FEDERICI, Apollinare: «Sitque imperante domino piissimo perpetuo augusto Octoni a deo coronato, pacifico, magno imperatore anno regni pietatis eius in Italia vero anno duodecimo, et domino Ottona item a deo coronato imperante eius filio anno sexto, die undecima mensis madii, indictione prima, Ravennen (del 973), id.: «Sitque imperante piissimo perpetuo augusto Ottone [II] ha deo coronato pacifico magno imperatore in Italia vero anno decimo, die trigesima iulii, indictione quinta, Ravenne» (del 977), etc. Les combinacions, com es pot veure, són variades; la repetició de anno hi és molt frequient. Les dates dels anys de Crist són les deduides pels editors, que sovint no sembla pas que corresponguin a les veritables; és clar que tot depèn del còmput cronològic preferit. 
Sigui com sigui, la indicció quinta, clarament conservada, correspon a l'anno primo d'Otó I, coronat emperador a Roma pel febrer del 962 . Aquesta correspondència entre l'any primer $i$ la indicció cinquena exclou la possibilitat que es tracti dels altres dos Otons: el segon, coronat emperador, en vida del pare, el 967 (indicció 10), i el tercer, coronat el 996 (indicció 9).

D'aquesta manera podem restituir el nom del papa mencionat a la primera línia del protocol, que seria sens dubte Joan XII abans de les enemistats amb l'emperador. Aquesta menció no ha d'estranyar en un document de Ravenna del segle $\mathrm{X}$, ja que la ciutat, d'obediència papal $\mathrm{i}$ a cens del pontífex romà, era al mateix temps lloc de pas dels nous emperadors germànics, els Otons, que reafirmaven per aquells anys llur domini senyorial sobre la península italiana i mantingueren a Ravenna la seva estança i cort més d'una vegada. 